\title{
Evaluation of intraoperative magnetic resonance imaging/ultrasound fusion optimization for low-dose-rate prostate brachytherapy
}

\author{
Stephen Abel, MScl, Paul Renz, DO², Olivier Gayou, PhD², Jie Tang, MSc², E Day Werts, PhD2,3, \\ Mark Trombetta, MD2,4 \\ 'Lake Erie College of Osteopathic Medicine, Bradenton, FL, ²Division of Radiation Oncology, Allegheny General Hospital, \\ Allegheny Health Network, Pittsburgh, PA, ${ }^{3}$ Temple University College of Medicine, Pittsburgh campus, Pittsburgh, PA, \\ ${ }^{4}$ Drexel University College of Medicine, Pittsburgh campus, Pittsburgh, PA, USA
}

\begin{abstract}
Purpose: Intraoperative planning with transrectal ultrasound (US) is used for accurate seed placement and optimal dosimetry in prostate brachytherapy. However, prostate magnetic resonance imaging (MRI) has shown superiority in delineation of prostate anatomy. Accordingly, MRI/US fusion may be useful for accurate intraoperative planning. We analyzed planning with MRI/US fusion to compare differences in dosimetry and volume to that derived from the postoperative computed tomography (CT).

Material and methods: Twenty patients underwent preoperative prostate MRI, which was fused intraoperatively with US during prostate brachytherapy. Intraoperative ${ }^{125} \mathrm{I}$ or ${ }^{103} \mathrm{Pd}$ seed placement was modified by the use of MRI fusion when indicated. Following implantation, dose comparisons were made between data derived from MRI/US and that from post-operative CT scans. Plan parameters analyzed included the $\mathrm{D}_{90}$ (dose to $90 \%$ of the prostate), rectal $D_{30}, V_{30}$ (volume of the rectum receiving 30 percent of dose), and prostate $V_{100}$.

Results: The median number of seeds implanted per patient was seventy-six. The MRI measured prostate volume, which was on average $4.47 \mathrm{cc}$ larger than the CT measured prostate volume. In 9 patients, the apex of the prostate was better identified under MRI with the fusion protocol, and an average of 4 fewer seeds were required to be placed in the apex/urinary sphincter region. Both MRI and US individually showed a reduced intraoperative prostate $D_{90}$ in comparison to the postoperative CT, with a larger mean difference for MRI in comparison with US (9.71 vs. 4.31 Gy, $p=0.007)$. This was also true for the prostate $\mathrm{V}_{100}(5.18 \mathrm{vs} .2 .73 \mathrm{cc}, p=0.009)$. Post-operative CT underestimated rectal $\mathrm{D}_{30}$ and $\mathrm{V}_{30}$ in comparison to both MRI and US with MRI showing a larger mean difference than US for $\mathrm{D}_{30}$ (40.64 vs. $35.92 \mathrm{~Gy}, p=0.04)$ and $\mathrm{V}_{30}(50.20$ vs. $44.38 \mathrm{cc}, p=0.009)$.

Conclusions: The MRI/US fusion demonstrated greater prostate volume compared to standard CT/US based planning likely due to the better resolution of the prostate apex. Furthermore, rectal dose was underestimated with CT vs. MRI based planning. Additional study is required to assess long-term clinical implications of disease control and effects on long-term toxicity, especially as related to the rectum and urinary sphincter. MRI/US intraoperative fusion may improve prostate dosimetry while sparing the rectum and urethra, potentially impacting disease control and late toxicity.
\end{abstract}

Key words: brachytherapy, dosimetry, MRI, prostate cancer.

\section{Purpose}

Prostate adenocarcinoma is the most commonly diagnosed cancer among American males, affecting approximately $15 \%$ of the population [1]. Screening measures such as rectal examination in combination with serum prostate-specific antigen (PSA) have made detection of cancer at an early, localized stage possible. When local- ized to the prostate, 10-year survival rates often exceed $95 \%$, highlighting the prolonged natural course of the disease [1]. Given the extended period of survival, complications relating to treatment can negatively impact the quality of life, contributing significantly to morbidity.

An excellent treatment option for men with early-intermediate stage disease is to undergo definitive treatment with brachytherapy. Low-dose-rate (LDR) brachytherapy 
involves the percutaneous implantation of permanent radioactive seeds into the prostate, and offers several advantages to prostatectomy including expedited recovery times, lower infection rates, and dramatically lower impotence rates [2].

Low-dose-rate prostate brachytherapy success is dependent on accurate visualization of the prostate gland. Inaccurate seed placement may result in dose heterogeneity, i.e., underdosing of tumor or overdosing either the prostate gland itself or organs at risk (OAR) such as the rectum and urethra. Adverse effects secondary to treatment can be analyzed both temporally (acute verse chronic), as well as by system (urinary system, gastrointestinal, sexual) [3]. Occurring at an incidence of approximately $10 \%$, the most common acute adverse effect is urinary retention [4]. Furthermore, up to $39 \%$ of post-implant patients report diminished sexual function as a result of treatment [5].

The current standard imaging modality for accurate seed placement and optimal dosimetry is intraoperative transrectal ultrasound (TRUS). While inexpensive and accessible, the use of TRUS is also highly operator-dependent; increasing the possibility of variance and subsequent error if the operator is inexperienced. Additionally, the ability of US to delineate prostate anatomy is inferior to that of alternative imaging such as magnetic resonance imaging (MRI).

Images obtained via MRI have consistently shown better soft tissue visualization of the intraprostatic tumor and better anatomic demarcation of the prostatic apex, external urinary sphincter, bladder neck, and intraprostatic ejaculatory ducts $[4,5,6,7,8,9]$. Over the past decade, clinicians have increasingly used MRI in prostate cancer evaluation and treatment decision making due to its improved image quality. As an example, Lawrentschuk et al. demonstrated the value of MRI in detection of previously biopsy negative cancer in patients with elevated serum of prostate specific antigen (PSA) levels [10]. Successful application of MRI in diagnosis of prostate cancer has also been accomplished through image guided biopsies [11]. Additionally, high-spatial-resolution dynamic contrastenhanced MRI has shown potential as a staging modality distinctly demonstrating capsular infiltration [12].

MRI information has changed the American Joint Commission on Cancer (AJCC) stage from T2 to T3 disease resulting in downstream treatment recommendations for changes in nearly one-fifth of patients in some studies $[13,14]$. The first report of intraoperative MRI-guided prostate brachytherapy successfully employed MRI with dose-volume histogram analysis in nine patients [15]. As a result, MRI imaging in prostate brachytherapy has been increasingly utilized on several platforms: preoperatively for planning, intraoperatively for implant guidance, and post-implant assessment of dosimetric parameters $[16,17]$. Intraoperative MRI scanning is impractical, but fusion programs in modern computers have made MRI incorporation possible. Given the functional relationship between seed location and dose-toxicity, superior visualization of the prostate, and recent evidence of MRI as a feasible modality in both diagnosis and therapy, we investigated the role of intraoperative MRI in prostate brachytherapy. This retrospective analysis compared the dosimetric parameters and acute morbidity of twenty patients who underwent intraoperative MRI/US fusion to the current standard of US-based planning.

\section{Material and methods}

\section{Patient selection}

From January 2015 to December 2015, twenty patients underwent transperineal prostate brachytherapy following evaluation, which consisted of a history and physical examination and relevant laboratory testing. All patients underwent a baseline International Prostate Symptom Score (IPSS) survey prior to the procedure and at all follow-up. Informed consent was obtained along with institutional review board approval. Eligibility criteria for participation in this protocol was consistent with recommendations of the American Brachytherapy Society and included the following: life expectancy $>5$ years, clinical tumor stage T1b-T2c, Gleason score $\leq 10$, PSA $\leq 50 \mathrm{ng} / \mathrm{ml}$, and no pelvic lymph node involvement or distant metastasis [18]. A preoperative prostate MRI was obtained and fused intraoperatively with US using a MiM Symphony ${ }^{\circledR}$ treatment planning system (MiM Software; Cleveland, Ohio, USA).

\section{Treatment planning}

The preoperative 1.5 Tesla MRI and US volumetric studies were obtained 2 weeks prior to the implant procedure. The prostate, rectum, and urethra were contoured intraoperatively and a treatment plan was generated for each patient to achieve a prostate $\mathrm{D}_{90}$ dose of approximately 160 Gy. Dosimetry was calculated both intraoperatively and one month post-operatively using the MiM Symphony ${ }^{\circledR}$ software, which incorporates the American Association of Physicists in Medicine Task Group 43 guidelines [19], and the implant was performed in accordance with the National Institute of Standards and Technology 1999 guidelines for prostatic brachytherapy [20]. The fusion process is done within the MiM Symphony computer system and is proprietary.

\section{Radioactive seed implantation}

Radioactive source implantation was accomplished through a transperineal approach using encapsulated ${ }^{125} \mathrm{I}$ or ${ }^{103} \mathrm{Pd}$ seeds with mean radioactivity of 0.381 and $1.31 \mathrm{mCi}$, respectively. Sources used were linear Bard Brachysource model IS- $1271^{\circledR}$ for ${ }^{125} \mathrm{I}$ and Bard model PS-TS200K ${ }^{\circledR}$ for ${ }^{103} \mathrm{Pd}$ (Bard Medical, Murray Hill, New Jersey, USA). Implantation was accomplished under guidance of MRI/ultrasound image fusion according to the preoperative treatment plan (Figure 1). Care was taken to exempt seeds from the proximal $0.5 \mathrm{~cm}$ surrounding the urethra, and $0.2-0.3 \mathrm{~cm}$ from the posterior capsule to protect the rectum. All patients were discharged the same day of the procedure, and were pre-emptively treated with tamsulosin $0.4 \mathrm{mg}$ daily and a broad-spectrum antibiotic post-implantation. The average prescribed dose to $90 \%$ of the target volume $\left(D_{90}\right)$ was 159.19 Gy for the 

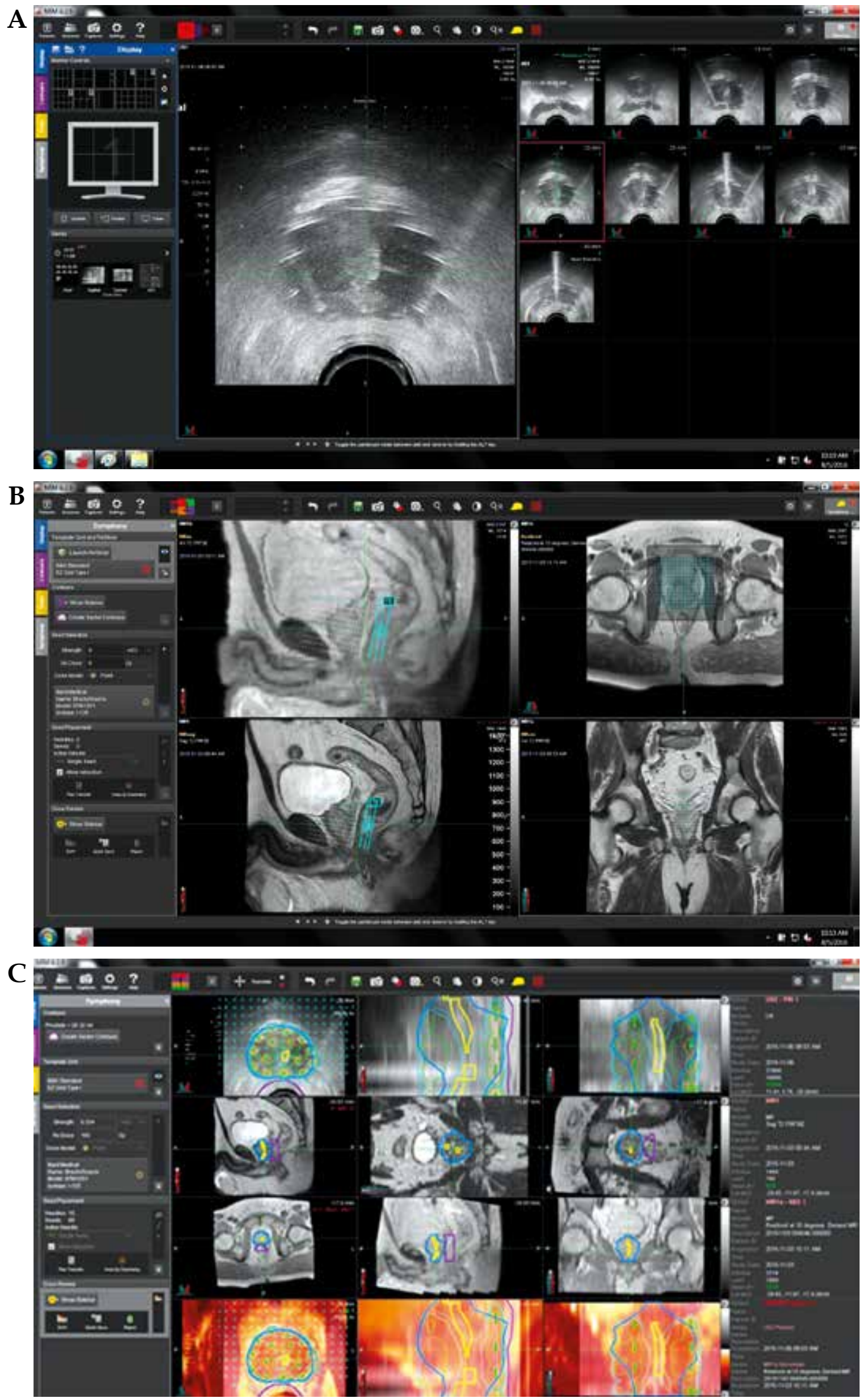

Fig. 1. Visualization of prostate using magnetic resonance imaging/ultrasound fusion imaging. A) Transrectal ultrasound of prostate gland, transverse view. B) Magnetic resonance imaging of prostate with ultrasound "slices" superimposed. C) Magnetic resonance imaging/ultrasound fusion 
${ }^{125}$ I patients, and 100.45 Gy for the ${ }^{103} \mathrm{Pd}$ patients based on post-operative computed tomography (CT) dosimetry done 4 weeks following the implant. The ${ }^{103} \mathrm{Pd}$ patients were treated as a boost following $45 \mathrm{~Gy}$ to the prostate using image guided radiotherapy (IMRT).

\section{Acute toxicity analysis}

Patients were evaluated for symptoms of urinary dysfunction using the IPSS [21]. The IPSS score rates patient reported symptoms 0-5, including: urgency, nocturia, weak stream, frequency, incomplete emptying, straining, and intermittency. Scores of $\leq 7$ are classified as "mild symptoms", between 8 and 19 as "moderate symptoms", and from 20 to 35 as "severe symptoms". Each patient completed an IPSS screening evaluation prior to and following implantation, and at each follow-up visit.

\section{Post-operative statistical analysis}

Following implantation, dosimetric comparisons were made between the preoperative MRI/US fusion, intraoperative US (without fused MRI), and post-operative CT scans. Plan parameters analyzed included the $D_{90}$ rectal $D_{30}$, rectal $V_{30}$ (volume of rectum receiving 30\% of the dose), and prostate $\mathrm{V}_{100}, \mathrm{~V}_{150}, \mathrm{~V}_{200}$. Patients completed a repeat IPSS survey at 2-3 weeks post-implantation that was compared to the results of their baseline survey. Unpaired t-tests were performed using Microsoft Excel $2013^{\circledR}$ (Microsoft Corporation, Bellevue, Washington, USA) software.

\section{Results}

Twenty patients underwent intraoperative MRI/ US fusion guided LDR brachytherapy with either ${ }^{125} \mathrm{I}$ or ${ }^{103} \mathrm{Pd}$. Characteristics relating to the patients and seed implants are listed in Table 1 . The median number of seeds implanted per patient was seventy-six. The MRI measured prostate volume was on average $4.47 \mathrm{cc}$ larger than the CT measured prostate volume. Prostate volumes and dosimetric characteristics measured through intraoperative Magnetic resonance imaging and US, as well as, post-operative CT are shown in Table 2.
In 9 patients, the apex of the prostate was better identified under MRI and an average of 4 fewer seeds (range, 3-5) were required to be placed in the apex/urinary sphincter region. All volumetric parameters demonstrated a mean reduction for MRI in comparison with CT vs. US in comparison with CT with largest mean reductions in the prostate $\mathrm{D}_{90}(9.71$ vs. $4.31 \mathrm{~Gy}, p=0.007)$, and $\mathrm{V}_{100}$ (5.18 vs. 2.73 cc, $p=0.009$ ) displayed in Table 3 . The mean difference in comparison with postoperative CT was larger for MRI vs. US in rectal $\mathrm{D}_{30}(40.64$ vs. $35.92 \mathrm{~Gy}$, $p=0.038)$, and $\mathrm{V}_{30}(50.20$ vs. $44.38 \mathrm{cc}, p=0.009)$.

\section{Discussion}

Low-dose-rate prostate brachytherapy is a definitive therapeutic option for localized disease that offers the advantage of being completed in an outpatient setting with relatively low acute morbidity. Consistent with past reports $[14,15,16]$, we have demonstrated that MRIbased prostate brachytherapy is a feasible option offering improved imaging and the potential for optimized seed placement and dose distribution. A minor limitation relating to MRI-based treatment is the increased intraoperative fusion time of 5-10 minutes and the introduction of pre-operative MRI imaging, which is rapidly becoming a standard preoperative study $[14,15,16,17]$. In addition to the slightly longer operative times, MRI is less accessible compared to US. Although more accessible, US does not offer the benefits of improved prostatic visualization and dosimetric parameters seen with MRI.

\section{Dosimetric parameters}

Several studies have validated prostate $D_{90}$ and $V_{100}$ as predictive measures of biochemical relapse-free survival (bRFS) [3,22,23,24,25]. Stock et al. [22] and Kollmeier et al. [23] demonstrated superior 8-year bRFS (82\% vs. $68 \%$ ) in patients treated with a cumulative $\mathrm{D}_{90} \geq 140 \mathrm{~Gy}$ compared to those treated with a dose < 140 Gy. Pötters et al. [24,25], found the 4-year bRFS in patients with $\mathrm{D}_{90}$ $\geq 90 \%$ to be better $(92.4 \%$ vs. $80.4 \%, p=0.001)$ compared to those who received $\mathrm{D}_{90}<90 \%$ of the prescription dose. Similarly Wallner et al. supported prostate $\mathrm{V}_{100}$ as a pre-

Table 1. Patient International Prostate Symptom Score and isotope characteristics

\begin{tabular}{lccc} 
Patient and implant characteristics & Median & Mean & Range \\
\hline Number of patients: $N=20$ & N/A & N/A & $(56-75)$ \\
\hline Age $(y)$ & 66.5 & 66.6 & $(1-19)$ \\
\hline IPSS score: pre-treatment & 9.5 & 8.5 & $(1-22)$ \\
\hline IPSS score: post-treatment & 12 & 11.4 & $(-8-17)$ \\
\hline Change in pre-treatment IPSS score & 3 & 3 & N/A \\
\hline Isotope: ${ }^{125}$ I $(n=19),{ }^{103} \mathrm{Pd}(n=1)$ & N/A & 78 & $(56-96)$ \\
\hline Number of seeds & 76 & 0.381 & $(0.309-0.439)$
\end{tabular}

IPSS - International Prostate Symptom Score, y-years, ${ }^{125}$ I - Iodine $125,{ }^{103} \mathrm{Pd}$ - Palladium 103 
Table 2. Comparison of dosimetric parameters obtained via intraoperative magnetic resonance imaging and ultrasound and post-operative computed tomography

\begin{tabular}{|c|c|c|c|}
\hline Dosimetric/volumetric parameters & MRI & US & Post-op CT \\
\hline \multicolumn{4}{|l|}{ Prostate volume (cc) } \\
\hline Mean & 37.8 & 36.58 & 33.34 \\
\hline Median & 36.11 & 35.5 & 30.74 \\
\hline Range & $(21.75-53.87)$ & $(21.75-58)$ & $(18.85-55.89)$ \\
\hline \multicolumn{4}{|l|}{$\mathrm{V}_{100}$ prostate $(\%)$} \\
\hline Mean & 85.25 & 87.7 & 90.43 \\
\hline Median & 85.62 & 89.92 & 90.49 \\
\hline Range & $(71.47-92.45)$ & $(74.55-93.06)$ & $(84.36-93.77)$ \\
\hline \multicolumn{4}{|l|}{$\mathrm{V}_{150}$ prostate $(\%)$} \\
\hline Mean & 25.32 & 26.01 & 45.97 \\
\hline Median & 24.02 & 23.79 & 45.81 \\
\hline Range & $(16.61-39.87)$ & $(17.42-43.82)$ & $(23.38-60.16)$ \\
\hline \multicolumn{4}{|l|}{$\mathrm{V}_{200}$ prostate $(\%)$} \\
\hline Mean & 10.82 & 10.97 & 23.07 \\
\hline Median & 9.84 & 9.68 & 21.62 \\
\hline Range & $(7.07-19.01)$ & $(6.99-21.22)$ & $(10.3-37.85)$ \\
\hline \multicolumn{4}{|l|}{$\mathrm{V}_{30}$ rectum $(\%)$} \\
\hline Mean & 66.74 & 59.54 & 17.76 \\
\hline Median & 71.66 & 61.11 & 17.24 \\
\hline Range & $(26.98-98.91)$ & (3.3-99.29) & $(3.44-36.02)$ \\
\hline \multicolumn{4}{|l|}{$\mathrm{D}_{90}$ prostate (Gy) } \\
\hline Mean & 149.48 & 154.87 & 159.19 \\
\hline Median & 152.46 & 159.97 & 161.35 \\
\hline Range & $(93.13-165.16)$ & $(87.83-166.65)$ & $(97.29-173.85)$ \\
\hline \multicolumn{4}{|l|}{$\mathrm{D}_{30}$ rectum (Gy) } \\
\hline Mean & 73.03 & 65.38 & 31.15 \\
\hline Median & 74.69 & 67.04 & 32.16 \\
\hline Range & $(37.21-122.36)$ & $(31.76-93.77)$ & $(6.45-54.12)$ \\
\hline
\end{tabular}

MRI - magnetic resonance imaging, US - ultrasound, CT - computed tomography, $V_{100}$ prostate - volume of prostate receiving 100 percent of prescribed dose $\left(V_{150}\right.$ and $V_{200}, 150 \%$ and $200 \%$, respectively), $V_{30}$ rectum - volume of rectum receiving $30 \%$ of prescribed dose, $D_{90}$ prostate - dose to $90 \%$ of prostate, $D_{30}$ rectum - dose to $30 \%$ of rectum

Table 3. Comparison of differences in dosimetric parameters between magnetic resonance imaging and computed tomography vs. ultrasound and computed tomography

\begin{tabular}{lccc} 
Mean dosimetric/Volumetric parameters & (CT-MRI) & (CT-US) & $p$-value \\
\hline Prostate volume (cc) & -4.47 & -3.24 & 0.281 \\
\hline $\mathrm{V}_{100}$ prostate $(\%)$ & 5.181 & 2.73 & 0.009 \\
\hline $\mathrm{V}_{150}$ prostate $(\%)$ & 20.65 & 19.95 & 0.073 \\
\hline $\mathrm{V}_{200}$ prostate $(\%)$ & 12.24 & 12.1 & 0.512 \\
\hline $\mathrm{V}_{30}$ rectum $(\%)$ & -50.2 & -44.38 & 0.009 \\
\hline $\mathrm{D}_{90}$ prostate $(G y)$ & 9.24 & 4.31 & 0.007 \\
\hline $\mathrm{D}_{30}$ rectum $(\mathrm{Gy})$ & -40.64 & -35.92 & 0.038
\end{tabular}

MRI - magnetic resonance imaging, US - ultrasound, CT - computed tomography, $V_{100}$ prostate - volume of prostate receiving 100 percent of prescribed dose $\left(V_{150}\right.$ and $V_{200}, 150 \%$ and $200 \%$, respectively), $V_{30}$ rectum - volume of rectum receiving $30 \%$ of prescribed dose, $D_{90}$ prostate - dose to $90 \%$ of prostate, $D_{30}$ rectum - dose to $30 \%$ of rectum 
dictive measure of bRFS [26]. Patients receiving $\mathrm{V}_{100}$ $\geq 90 \%$ of the prescription dose had superior 3-year bRFS ( $97 \%$ vs. $87 \%, p=0.01$ ) compared to those with $\mathrm{V}_{100}<90 \%$. Our median post-operative CT dosimetric parameters reported herein of $\mathrm{D}_{90}(161.35 \mathrm{~Gy})$ and $\mathrm{V}_{100}(90.49 \%)$ were within the ranges recommended to maximize bRFS. Both the $\mathrm{V}_{100}$ (median, 85.62\%) and $\mathrm{D}_{90}$ (median, $152.46 \mathrm{~Gy}$ ) were smaller when calculated using MRI-based planning. This decrement likely reflects better anatomic definition and subsequent increased contouring accuracy seen with MRI. While increasing prostate $V_{100}$ and $D_{90}$ that were correlated with increased bRFS, exceedingly high doses may lead to increased morbidity. Stock and Stone showed worsening late urinary complications when $\mathrm{D}_{90}>180 \mathrm{~Gy}$ [27]. Our $D_{90}$ dose (range, 97.29-173.85 Gy) was well below this threshold in all patients. Monitoring of the rectal $\mathrm{D}_{30}$ showed measurement discrepancies consistent with those noticed by other researchers in MRI/CT comparison [28]. We used this same parameter to demonstrate discrepancies with ultrasound defined volumes suggesting that caution must be used when evaluating dose to larger volumes of rectum (as opposed to 1 and 2 cc volumes only), and using these values as limits for organs at risk, since the $D_{30}$ has been shown as a measurable metric for long-term morbidity [29].

\section{Acute toxicity and quality of life}

In a study by Cesaretti et al., the average pre-treatment IPSS in 172 patients was 7.5 (CI, 6.5-8.5). This value was similar to the average pre-treatment IPSS (8.5) seen in our study [30]. They also observed that baseline IPSS scores increased after seed implantation, typically reaching a peak value of $19.4(\triangle \mathrm{IPSS}=11.9)$ at a median of 1.3 months post-implant. These results were similar to those previously obtained by Desai et al., in which IPSS also peaked at 1 month post-implant to 14 [31]. Increasing total radioactivity and cumulative radiation dose to the prostate, bladder, and urethra were variables correlated with the peak in symptoms. The IPSS scores of our patients were assessed at 3-4 weeks post-treatment, with a mean change from baseline IPSS of 3 . While this increase in IPSS compares favorably with the reported results of Desai and Cesaretti (9 and 11.9, respectively), our sample size was much smaller with the IPSS taken at an earlier post-operative interval, possibly contributing to the smaller incremental increase.

Prostate volume significantly impacts the IPSS score $[27,28,29,30,31,32,33]$. The average prostatic gland volume by ultrasound in our study (mean, $36.58 \mathrm{cc}$; range, $21.75-58.0 \mathrm{cc}$ ) was similar to volumes reported in both Desai (median, 32.6 cc; range, 16-72 cc) [31] and Cesaretti (mean, $42.9 \mathrm{cc}$; range, $12.7-73.7 \mathrm{cc}$ ) [30].

\section{Utility of magnetic resonance imaging}

MRI is increasingly used for prostate cancer screening, staging, and treatment. With this technique, we were able to visualize the prostatic apex more clearly. As a result, nearly half of the patients $(9 / 20)$ treated required fewer radioactive seeds in this area, which may result in decreased dose to the urogenital diaphragm and associated distal urethra. With improved visualization and dosimetry, we anticipate diminished acute and chronic morbidity. Despite the feasibility and superior dosimetric parameters seen with MRI imaging, additional larger scale studies are required to assess the long-term clinical implications of disease control and normal tissue morbidity.

\section{Conclusions}

The current standard planning modality in prostate brachytherapy is CT/US-based. Our study showed that CT/US-based planning may underestimate prostate volume when compared to treatment planning conducted with the MRI/US fusion. This may contribute to unnecessary total seeds implanted and to possible excessive total dose. Furthermore, CT/US-based dosimetric plans underestimated prostatic and rectal dose when compared to MRI/US, as well potentially contributing to increased morbidity. Further study is required to define the longterm clinical significance. Altogether, MRI is a feasible imaging option for prostate brachytherapy capable of institution into standard treatment protocols to augment intraoperative TRUS-based seed placement.

\section{Disclosure}

Authors report no conflict of interest.

\section{References}

1. Siegel R, Miller K, Jemal A. Cancer Statistics 2016. CA Cancer J Clin Oncol 2016; 66: 7-30.

2. Hyun JS. Prostate Cancer and Sexual Function. World J Mens Health 2012; 30: 99-107.

3. Lee WR. Permanent Prostate Brachytherapy: The Significance of Postimplant Dosimetry. Rev Urology 2004; 6 (Suppl 4): S49-S56.

4. Crook J, McLean M, Catton C et al. Factors influencing risk of acute urinary retention after TRUS-guided permanent prostate seed implantation. Int J Radiat Oncol Biol Phys 2002; 52: 453-460.

5. Beyer D. The evolving role of prostate brachytherapy. Cancer Control 2001; 8: 163-170.

6. Tanderup K, Viswanathan A, Kirisits C et al. Magnetic resonance image guided brachytherapy. Semin Radiat Oncol 2014; 24: 181-191.

7. Westphalen A, Mckenna D, Kurhanewicz J et al. Role of Magnetic Resonance Imaging and Magnetic Resonance Spectroscopic Imaging Before and After Radiotherapy for Prostate Cancer. J Endourol 2008; 22: 789-794.

8. Carroll PR, Coakley FV, Kurhanewicz J. Magnetic resonance imaging and spectroscopic imaging of prostate cancer. Rev Urol 2006; 8: S4-S10.

9. Milosevic M, Voruganti S, Blend R et al. Magnetic resonance imaging (MRI) for localization of the prostatic apex: comparison to computed tomography (CT) and urethrography. Radiother Oncol 1998; 47: 277-284.

10. Lawrentschuk N, Fleshner N. The role of magnetic resonance imaging in targeting prostate cancer in patients with previous negative biopsies and elevated prostate-specific antigen levels. BJU Int 2009; 103: 730-733.

11. Marks L, Young S, Natarajan S. MRI-ultrasound fusion for guidance of targeted prostate biopsy. Curr Opin Urol 2013; 23: 43-50. 
12. Bloch B, Furman-Haran E, Helbich T et al. Prostate Cancer: Accurate Determination of Extracapsular Extension with High-Spatial-Resolution Dynamic Contrast-enhanced and T2-weighted MR Imaging - Initial Results. Radiology 2007; 245: 176-185.

13. Edge SB, Byrd DR, Compton CC et al. AJCC cancer staging handbook. $7^{\text {th }}$ ed. Springer-Verlag, New York 2010.

14. Clarke D, Banks S, Wiederhorn A et al. The role of endorectal coil MRI in patient selection and treatment planning for prostate seed implants. Int J Radiat Oncol Biol Phys 2002; 50: 903-910.

15. D'amico AV, Cormack R, Tempany CM et al. Real-time magnetic resonance image-guided interstitial brachytherapy in the treatment of select patients with clinically localized prostate cancer. Int J Radiat Oncol Biol Phys 1998; 42: 507-515.

16. Sannazzari GL, Ragona R, Ruo Redda MG et al. CT-MRI image fusion for delineation of volumes in three-dimensional conformal radiation therapy in the treatment of localized prostate cancer. Br J Radiol 2002; 75: 603-607.

17. Ohashi T, Momma T, Yamashita S et al. Impact of MRI-based postimplant dosimetric assessment in prostate brachytherapy using contrast-enhanced T1-weighted images. Brachytherapy 2012; 11: 468-475.

18. Merrick GS, Zelefsky M, Sylvester Ket al. American Brachytherapy Society Prostate Low-Dose Rate Task Group. Availabel at: http://www.americanbrachytherapy.org/guidelines/prostate_low-doseratetaskgroup.pdf.

19. Rivard MJ, Coursey BM, DeWerd LA et al. Update of AAPM Task Group No. 43 Report: A revised AAPM protocol for brachytherapy dose calculations. Med Phys 2004; 31: 633-674.

20. Beyer D, Nath R, Butler W et al. American Brachytherapy Society recommendations for clinical implementation of NIST1999 standards for ${ }^{103}$ palladium brachytherapy. Int J Radiat Biol Oncol Phy 2000; 47: 273-275.

21. Barry MJ, Fowler FJ Jr, O'Leary MP et al. The American Urological Association symptom index for benign prostatic hyperplasia. The Measurement Committee of the American Urological Association. J Urol 1992; 148: 1549-1557.

22. Stock RG, Stone NN, Tabert A et al. A dose-response study for I-125 prostate implants. Int J Radiat Oncol Biol Phys 1998; 41: 101-108.

23. Kollmeier MA, Stock RG, Stone N et al. Biochemical outcomes after prostate brachytherapy with 5-year minimal follow-up: importance of patient selection and implant quality. Int J Radiat Oncol Biol Phys 2003; 57: 645-653.

24. Potters L, Cao Y, Calugaru E et al. A comprehensive review of CT-based dosimetry parameters and biochemical control in patients treated with permanent prostate brachytherapy. Int J Radiat Oncol Biol Phys 2001; 50: 605-614.

25. Potters L, Huang D, Calugaru E et al. Importance of implant dosimetry for patients undergoing prostate brachytherapy. Urology 2003; 62: 1073-1077.

26. Wallner K, Merrick G, True L et al. 125I versus 103Pd for lowrisk prostate cancer: preliminary PSA outcomes from a prospective randomized multicenter trial. Int J Radiat Oncol Biol Phys 2003; 57: 1297-1303.

27. Stock RG, Stone NN. Importance of post-implant dosimetry in permanent prostate brachytherapy. Eur Urol 2002; 41: 434-439.

28. Tanaka O, Hayashi S, Matsuo M et al. Comparison of MRIbased and CT/MRI fusion-based post implant dosimetric analysis of prostate brachytherapy. Int J Radiat Oncol Biol Phys 2006; 66: 597-602.

29. Shiraishi Y, Yorozu A, Ohashi T et al. Dose constraint for minimizing grade 2 rectal bleeding following brachytherapy combined with external beam radiotherapy for localized prostate cancer: rectal dose-volume histogram analysis of 457 patients. IntJ Radiat Oncol Biol Phys 2011; 81: e127-133.
30. Cesaretti JA, Stone NN, Stock RG. Urinary symptom flare following I-125 prostate brachytherapy. Int J Radiat Oncol Biol Phys 2003; 56: 1085-1092.

31. Desai J, Stock RG, Stone NN et al. Acute urinary morbidity following I-125 interstitial implantation of the prostate gland. Radiat Oncol Invest 1998; 6: 135-141.

32. Merrick GS, Butler WM, Lief JH et al. Temporal resolution of urinary morbidity following prostate brachytherapy. Int J Radiat Oncol Biol Phys 2000; 47: 121-128.

33. Keyes M, Miller S, Moravan V et al. Predictive factors for acute and late urinary toxicity after permanent prostate brachytherapy: long-term outcome in 712 consecutive patients. Int J Radiat Oncol Biol Phys 2009; 73: 1023-1032. 